\title{
Acquired resistance of malarial parasites against artemisinin-based drugs: social and economic impacts
}

\author{
Johanna M Porter-Kelley' \\ Joann Cofie ${ }^{2}$ \\ Sophonie Jean ${ }^{2}$ \\ Mark E Brooks' \\ Mia Lassiter' \\ DC Ghislaine Mayer ${ }^{2}$ \\ 'Life Sciences Department, \\ Winston-Salem State University, \\ Winston Salem, NC, USA; \\ ${ }^{2}$ Department of Biology, Virginia \\ Commonwealth University, \\ Richmond, VA, USA
}

This article was published in the following Dove Press journal:

Infection and Drug Resistance

26 August 2010

Number of times this article has been viewed

\begin{abstract}
Malaria, a disease of poverty and high morbidity and mortality in the tropical world, has led to a worldwide search for control measures. To that end, good antimalarial chemotherapies have been difficult to find in the global market and those that seem to be most effective are rapidly becoming ineffective due to the emergence and spread of drug resistance. Artemisinin, a very effective yet expensive antimalarial, has quickly become the recommended drug of choice when all other possibilities fail. However, for all its promise as the next great antimalarial, the outlook is bleak. Resistance is developing to artemisinin while another effective antimalarial is not in sight. Malaria endemic areas which are mostly in developing countries must deal with the multifaceted process of changing and implementing new national malaria treatment guidelines. This requires complex interactions between several sectors of the affected society which in some cases take place within the context of political instability. Moreover, the cost associated with preventing and containing the spread of antimalarial resistance is detrimental to economic progress. This review addresses the impact of artemisinin resistance on the socioeconomic structure of malaria endemic countries.
\end{abstract}

Keywords: artemisinin-based drugs, social, economic, malarial parasite resistance

Malaria, a mosquito-borne parasitic disease, affects 510 million people causing almost 3 million deaths annually. ${ }^{1}$ For more than 50 years, cinchona alkaloids and their derivatives were an integral part of malaria control. Indeed, chloroquine was one of the most effective synthetic antimalarial drugs ever produced. However, chloroquine-resistance falciparum malaria was first reported in South East Asia and South America and has now spread through Asia, Africa, and South America. ${ }^{2}$ This has led to a global resurgence of malaria. Currently, these alkaloids are being replaced by the terpene Artemisia annua, also known as qing hao or sweetworm, whose active ingredient is artemisinin. Discovered in 1971 by Chinese scientists, artemisinin was introduced to the world in 1979. ${ }^{3}$ Although quite expensive to produce, artemisinin is one of the most effective antimalarials. It is currently grown by farmers in China, Vietnam, and parts of Africa. With a long growing season, the process of extracting artemisinin from the leaves is complex and time consuming. Because of the difficulty in extracting it from the plant, a semi-synthetic version generated by the addition of chemical groups to extracted artemisinin is commonly used. Dihydroartemisinin (DHA) is the product of the first step; additional synthetic steps give rise to artesunate, artemether, and arteether which are metabolized back to dihydroartemisinin in the body (Figure 1). The artemisinin derivatives possess greater antimalarial properties. They are active against multiple drug-resistant parasites and have a very low level
Correspondence: DC Ghislaine Mayer Department of Biology, Virginia Commonwealth University, 1000 W. Cary St., Rm. 126, Richmond, VA 23284-2012, USA

Tel + I 804-828-0828

Fax +l 804-828-0503

Email gmayer@vcu.edu 


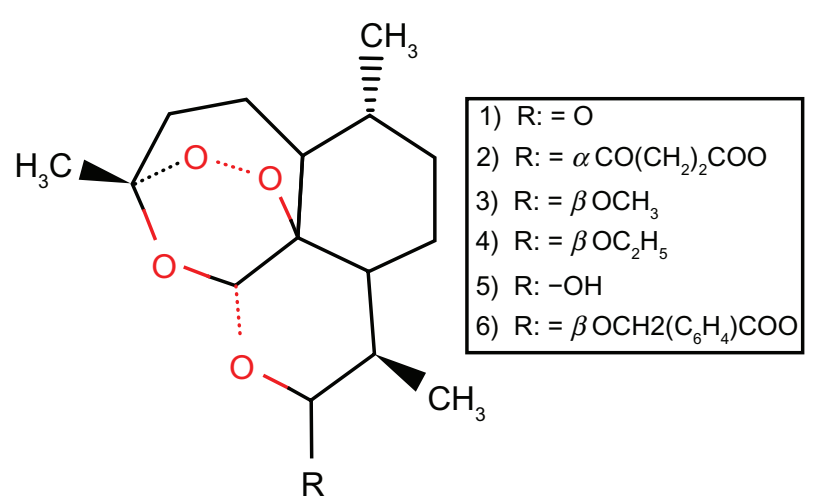

Figure I Chemical structure of artemisinin and derivatives. Diagram of artemisinin and its derivatives. I, artemisinin; 2, artesunate; 3, artemether; 4, arteether; 5, dihydroartemisnin; 6, artelinic acid.

of toxicity to humans. As an antimalarial drug, artemisinin has a broad range of action against different stages of the parasite. It kills young circulating ring-stage parasites as well as the mature stages that sequester on blood vessels. ${ }^{3}$ Because of these properties, artemisinin is very useful in cases of severe malaria. Because of its broad range of action, dispensation of a single dose kills a large number of parasites. All the stages of the $P$. falciparum life cycle, including the gametocytes which transmit the infection to mosquitoes, are killed by artemisinin. Artemisinin is known to decrease the rate of gametocyte carriage and gametocyte density in a population. ${ }^{3-5}$

Although very little is known about the molecular mechanism of action of artemisinin and its derivatives, there are a few studies that give insight into its mechanism. For instance, after treatment of $P$. berghei-infected mice and $P$. falciparum parasites grown in human erythrocytes with artemisinin, trophozoites showed morphological changes, such as swelling and spiral deformation of the membrane of the food vacuole when blood samples were examined by electron microscopy. ${ }^{5}$ Results of additional studies have shown that artemisinin is a blood schizontocide that has an inhibitory action on the parasite's protein and nucleic acid metabolism. ${ }^{6}$ While the mechanism of action of artemisinin has not been elucidated, it was recently revealed that the endoperoxide bridge is essential for arteminsinin killing (Figure 1). The sarcoplasmic endoplasmic reticulum calcium adenosine triphosphatase (PfATPase 6) has been promulgated as the main target. ${ }^{7}$ This assessment is based on mutational studies in the gene coding for PFATPase 6. P. falciparum parasites from French Guyana with point mutations in the gene encoding PfATPase 6 showed resistance to artemisinin derivatives. ${ }^{8}$ Nevertheless, these findings have not been confirmed in parasites from other parts of the world.

\section{Malaria drug resistance}

The reemergence of malaria drug resistance is a major problem in the tropical and subtropical world. Several factors influence the reemergence of drug resistance. Among them is the level of immunity against the malaria parasite in the population. ${ }^{9}$ For instance, in low or unstable transmission areas, drug resistance propagates rapidly. This is due to minimal immunity in a population, thus parasite infections lead to acute symptomatic disease which is most likely treated. Therefore, drug resistance is likely to propagate rapidly due to high drug pressure on existing parasites. In fact, certain mutations are prevalent as a result of high drug pressure in most sub-Saharan countries. ${ }^{10}$ On the other hand, in areas where malaria transmission is high, the spread of drug resistance is restricted because of high levels of immunity. Here, there is less need for treatment in a population because there are fewer clinical symptoms. One study based in Tanzania showed that immunity enhanced drug efficacy. ${ }^{10}$

\section{Changing national malaria treatment policy}

Malaria parasite resistance is a complex process that requires constant monitoring and strategic actions to save lives. New national malaria treatment policies have been slow to implement at the country level and therefore have not been very effective in relieving the burden of disease. Many malaria endemic countries have relied on chloroquine as a first-line of treatment against malaria for several decades. Chloroquine was the antimalarial drug of choice because of its low cost and efficacy. The spread of chloroquine resistance across malaria-endemic areas has caused an incredible challenge for the majority of endemic countries. ${ }^{11-14}$ National malaria control programs of endemic countries had to develop malaria treatment policies in response to elevated levels of drug resistance to chloroquine (CQ) and sulphadoxine-pyrimethamine (SP). ${ }^{15-19}$ This has led to an international effort to replace CQ with relatively more expensive but highly effective artemisinin-based combination therapies (ACTs). The drugs containing artemisinin show $100 \%$ effectiveness after a three to five day regimen. However, due to the intensive labor and length of production, these drugs are costly, making them inaccessible to the world's poorest countries. ${ }^{19}$

A change in international therapeutic recommendations does not always translate to an immediate policy change at country levels. ${ }^{14,20-22}$ According to the World Health Organization (WHO), all African countries have switched from using chloroquine, except Cape Verde and Eritrea for treatment 
of falciparum malaria. ${ }^{23}$ In Cape Verde, SP is used when chloroquine fails. Likewise, the Maldives Islands of South East Asia continue to use chloroquine-based antimalarial therapies with recourse to a combination of mefloquine and SP when treatment fails. ${ }^{23}$ Most malaria endemic countries have entered into partnership with the World Health Organization (WHO) Roll Back Malaria program since 1998 and have thus made a concerted effort to control malaria. ${ }^{24,25}$ The result of this effort was the enactment through National Malaria Control Programs with recommendation of new antimalarials as first-line treatments. Since 1998, new policy suggested that malaria endemic areas change their firstline recommendations for the treatment of malaria at least twice before advocating the use of artemisinin-combination therapies. ${ }^{21,22}$ A good example of a country changing its policies is Tanzania. There, malaria is most commonly a disease of rural agricultural communities, and widespread antimalarial drug resistance has been reported. ${ }^{12}$ Although a plan was initiated in Tanzania, the number of cases and deaths due to malaria are still increasing; this increase is thought to be due to resistance to antimalarial drugs. Chloroquineresistant falciparum malaria is widespread in Tanzania, and sulfadoxine-pyrimethamine (SP) was only introduced in 2001. Despite this, resistance to SP has been found in a number of areas. The first-line treatment was changed again to artemisinin-based combination therapy in $2006 .{ }^{14}$

\section{Artemisinin resistance}

Artemisinin is currently playing an important role in the control of falciparum malaria. However, CQ and SP, used singly or in combination, remain the drugs of choice for the treatment of vivax malaria. ${ }^{26}$ Artemisinin has a very short half-life and quickly clears from the body by glycoronidation, with CYP 2B6, the primary catalyst. ${ }^{27,28}$ This characteristic renders it useless as a prophylaxis. ${ }^{6}$ However, it gave credence to the hypothesis that mutations will not arise against artemisinin very easily. Though artemisinin and its derivatives were initially used as monotherapies, they are now used in combination because of the high rate of emergence of resistance against several antimicrobial drugs given as monotherapies. Artemisinin is often given in combination with a variety of antimalarials including those with a longer half-life. Indeed, artemisinin combination therapy (ACT) is now the suggested treatment for falciparum malaria in all endemic areas. In 2006, the World Health Organization (WHO) issued guidelines for the treatment of malaria and recommended ACT as the first-line of treatment in all endemic areas. ${ }^{3}$ The recommendations for global use of artemisinin derivatives were founded on their outstanding tolerability, safety, and efficacy. With no alternative drugs, artemisinin was to be protected from resistance by controlled rational use with the development and implementation of global policies. During a meeting of the WHO that took place in 1998 to review the use of artemisinin, the experts gave advice on the appropriate use of artemisinin in different epidemiological situations. ${ }^{29} \mathrm{~A}$ report was generated outlining detailed recommended drug regimens, clinical use, and priority areas for research. ${ }^{29}$ Drug regimens and clinical use were outlined for each country. A summary of the recommendations is shown in Table 1. Malaria endemic countries with the assistance of WHO have carefully developed and implemented policies aimed at controlling the use of artemisinin. It was recommended that public health services be the sole means of allowing artemisinin in the market.

Long before these efforts to prevent resistance against artemisinin, resistance was first reported in Pailin, Cambodia in the $1970 \mathrm{~s} .{ }^{30}$ This city has been the site of three successive waves of antimalarial resistance. Here, resistance first developed against chloroquine then sulfadoxine-pyrimethamine. ${ }^{3,31}$ Resistant parasites have extended not only throughout Cambodia and Asia but also to sub-Saharan Africa, rendering important weapons in the fight against falciparum malaria ineffective. ${ }^{3}$ Antimalarial resistance is defined as the increased parasite clearance time following treatment whereas treatment failure is defined as the presence of fever and parasitemia after three days of treatment. It normally takes three days for parasites to clear in patients after drug treatment. $^{3}$ The first official report about the decreasing effectiveness of artemisinin was published by WHO in 2005. ${ }^{32}$ In addition to four provinces in Cambodia, $P$. falciparum parasites are also believed to be resistant in two provinces in Thailand. ${ }^{31}$ Countries such as Cambodia, Myanmar, and Vietnam, which have allowed artemisinin into the market

Table I Recommended artemisinin combination therapies. Adapted from Roll Back Malaria Partnership: Facts on ACTs January 2006 Update

\begin{tabular}{ll}
\hline Antimalarial combinations & Restrictions \\
\hline $\begin{array}{l}\text { Artemether/lumefantrine } \\
\text { Artesunate with amodiaquine }\end{array}$ & $\begin{array}{l}\text { In areas where the cure rate of } \\
\text { amodiaquine monotherapy is greater } \\
\text { than } 80 \%\end{array}$ \\
Artesunate with mefloquine & $\begin{array}{l}\text { Insufficient safety data to recommend } \\
\text { its use in Africa }\end{array}$ \\
Artesunate with sulfadoxine/ & $\begin{array}{l}\text { In areas where the cure rate of } \\
\text { sulfadoxine/pyrimethamine is } \\
\text { pyrimethamine }\end{array}$ \\
\hline
\end{tabular}


without the supervision of the public health services, have tremendous problems with resistance. In fact, artemisinin has been used as a monotherapy in Western Cambodia for nearly 30 years. In 2009, scientists confirmed the emergence of $P$. falciparum parasites that have developed resistance to artemisinin derivatives in Pailin, Cambodia. This resistance has now spread to other cities of Cambodia as well as to the Thai-Myanmar border. In order to combat this dilemma WHO has suggested that countries employ the use of ACTs, to lower the risk of producing more artemisinin-resistant Plasmodium parasites. $^{24}$

Artemisinin combination therapies (ACTs) are a combination of an artemisinin derivative with another antimalarial such as piperaqine or amodiaquine. A list of artemisinin-based therapies is shown in Table 1. In 2001, WHO launched its "Roll Back Malaria Partnership", a campaign that outlined the use of artemisinins in conjunction with medications such as lumefantrine, amodiaquine, mefloquine, sulfadoxine, or pyrimethamine. ${ }^{24} \mathrm{~A}$ list of antimalarial drugs and their abbreviations can be found in Table 2. As of 2006, 56 countries in Africa, Asia, and South America adopted the use of WHO guidelines for treating malarial infections caused by Plasmodium falciparum with ACTs. ${ }^{24}$ In 2007, the number of countries in Africa using ACTs increased by 41. The impact of ACT treatment on malaria transmission may vary among artemisinin combinations although the effect is synergistic. $^{33}$

Artemisinin has played a pivotal role in the control of malaria worldwide. The unwelcome possibility that Plasmodium parasites can develop complete resistance to artemisinin derivatives could be a catastrophe of monumental proportion for global health. Currently, there are no drugs in the pipeline to replace artemisinin, and it could take five or more years to find a replacement drug. ${ }^{3,34}$ The widespread resistance of Plasmodium parasite to artemisinin would spell disaster for sub-Saharan African countries that had made significant gain in reducing morbidity and mortality due to malaria.

Intervention efforts spearheaded by WHO, the national malaria control programs, and the Gates foundation to contain the spread of artemisinin resistance are currently underway. ${ }^{35}$ The primary approach in containing the spread of artemisinin resistance is to control the sale of this antimalarial in the private sector. This involves campaigns by the governmental agencies to inform the private sectors, which include pharmacies and shops, about the ban against artemisinin monotherapy. The removal of artemisinin monotherapy from the market will greatly reduce the pressure of a single drug on the parasite and cause them to revert
Table 2 Abbreviations of currently used antimalarial drugs

\begin{tabular}{ll}
\hline AE & Arteether \\
AQ & Amodiaquine \\
AL & Artemether-lumefantrine \\
AM & Artemether \\
ART & Artemisinin \\
AS & Artesunate \\
CL & Clindamycine \\
CQ & Chloroquine \\
D & Doxycycline \\
DHA & Dihydroartemisinin \\
MQ & Mefloquine \\
NQ & Naphroquine \\
PG & Proguanil \\
PM & Pyrimethamine \\
PPQ & Piperaquine \\
PQ & Primaquine \\
PYR & Pyronaridine \\
QN & Quinine \\
SP & Sulfadoxine-pyrimethamine \\
T & Tetracycline \\
TRI & Trimethoprim \\
\hline
\end{tabular}

to the wild type genotypes which are genetically more fit. The campaigns take the form of workshops and radio and television broadcasts. ${ }^{33,35}$ Law enforcement agencies are also involved in the effort. Their role is to make sure that the ban is enforced. Moreover, a concerted effort to remove antimalarial drugs from the local stores and to allow trained villagers to dispense the malaria drugs after proper diagnostic tests have been performed is underway. ${ }^{33,35}$ This insures that every case of fever is not treated with antimalarials. It is possible that this strategy may not be successful as it is dependent on infected individuals displaying symptoms. In endemic areas, many individuals, especially adults, are asymptomatic and carry a low parasite load. These asymptomatic individuals would still able to transmit artemisinin-resistant parasites. To counteract this, there are programs in place in Cambodia to screen and treat all patients who are positive for infection with drugs regardless of symptoms. ${ }^{35}$ In addition, efforts are also aimed at prevention by intensifying the wide scale distribution of bednets. A population dynamic mathematical modeling framework was developed to explore the relative effectiveness of a variety of containment interventions in eliminating artemisinin-resistant malaria in western Cambodia. ${ }^{36}$ The most effective intervention to eliminate artemisinin-resistant malaria was a switch of treatment from artemisinin monotherapy to ACT with 4 years being the average estimated time for elimination of the resistant parasites. However, with this approach it is predicted that elimination of artemisinin-resistant malaria using ACT can be achieved only by elimination of all malaria cases in the area. 
In this case various forms of ACT are more effective against infections with artemisinin-sensitive parasites, leaving the more resistant infections at an increasing proportion of the dwindling parasite population. ${ }^{36,37}$ That in and of itself is a daunting task. The emergence of artemisinin in South East Asia and the potential spread to all malaria endemic areas would pose both social and economic challenges for these countries.

\section{Social impact of artemisinin resistance}

The first change in malaria treatment in endemic areas involved shifting from the failing inexpensive CQ to SP, another inexpensive monotherapy. ${ }^{23,25}$ The spread of antimalarial resistance with the resulting challenges of implementing national malaria drug policies occurs in social contexts where self-medication, mistrust of government officials, and lack of compliance are common. The changes are introduced by the Ministry of Health of various governments which are often not trusted. In Tanzania, this took place in 2001. ${ }^{14}$ There was some reluctance on the part of many to use SP when it was first introduced to replace CQ. ${ }^{14,18}$ Because CQ was used for several decades and was considered to be safe and effective, many households in Tanzania still had stockpiles of CQ. Moreover, health care practitioners there had developed a certain attachment to $\mathrm{CQ}$. To make matters worse, mothers of sick children felt they were being forced to abandon CQ which they considered to be more effective than SP because the recommended dose of the new drug did not quickly relieve fever, the outward symptom of malaria. This led to not only suspicion and noncompliance, but a continued use of $\mathrm{CQ}$, the failing drug. ${ }^{21}$

Furthermore, the acceptance of new antimalarials by a community is dependent on the degree of knowledge about the new drug and its proper use. Most individuals are informed by health care practitioners or by materials displayed at health care facilities. In some cases, the media by way of television, and commonly radio and newspapers, play an important role in disseminating information about the new antimalarials. This can lead to fear since oftentimes the journalists emphasize the negative side effects of the new drugs. Successful transition to new antimalarials is strongly dependent on journalists being educated about the new drug in order to disseminate correct information to the general public. Once a negative opinion is formed about a drug, the general public develops a prejudice against it and it is a major challenge to accept the new antimalarial despite its effectiveness. It has been reported that mothers would not give the new antimalarials because of their perceived ill effects. ${ }^{18,21}$ They would go as far as inducing vomiting in their children to prevent them from consuming the new drug. This puts increased pressure on health care workers who have to closely monitor patients to ensure ingestion of the drug before being allowed to return to their home. In addition, changes to new drugs are always accompanied by anxieties on the part of the public regarding perceived or experienced adverse reactions. Although few individuals had experienced adverse reactions, every person interviewed had learned through the media about deaths following the use of SP. ${ }^{21}$

When SP started to fail in 2003, introduction of artemisinin combination therapies (ACTs) was met with even greater resistance by members of the community, especially caregivers, because ACTs were not made available over the counter like chloroquine. ${ }^{18,21}$ It is very likely that similar reactions would be observed if a new regimen of antimalarials were to be recommended so soon after switching to ACT. It was expected that ACT would have a lifespan as long as chloroquine. The rise and spread of acquired resistance to artemisinin-based therapy might erode the trust of the population in the ability to provide a long-lasting drug against malaria. There were already thoughts of government conspiracy aimed at killing off the population or experimenting on the population with new antimalarials from some participants in a focus discussion group aimed at assessing the perception of various social groups in Tanzania about the recommendation of SP as the new first-line of treatment against malaria. ${ }^{21}$

\section{Economic impact of malaria}

In our consideration of the economic impact of artemisinin resistant malaria, we first consider the impact of malaria itself on a country's economy. We will then consider the impact from the use of artemisinin. Lastly, we consider the economic impact of artemisinin-resistant malaria. ${ }^{38}$

First, we acknowledge that there are many factors that have an impact on a country's economy other than malaria. Some of these factors that result in low incomes and low economic growth are poor soils, low agricultural productivity, tropical diseases other than malaria, trade barriers, weak institutions, poor economic policies, ethnic conflict, and the residue of colonization. ${ }^{38}$ Although all these factors play a role in the economy of a country there are reports that consider the economic impact of malaria to be so high that it is considered the major cause of poverty. ${ }^{39}$

The economic impact of malaria takes a toll on the population living in an endemic area. It is actualized in individual as well as government costs. To an individual, the 
cost can accumulate in the expense of medical treatment, travel to dispensaries and clinics, preventions, and burial upon death, as well as loss of income from days away from work and school. Malaria has even been implicated in learning disability in children by which the far reaching effects cannot be realized and calculated in the effect of malaria on an economy. ${ }^{39}$ On the other hand, governments sustain costs in health care facility maintenance, drugs and supplies, insecticide spraying, and bednet distributions. They also sustain loss of income from sick workers, economic ventures, and lack of tourism. For example, peak periods of malaria transmission often coincide with the peak period of agricultural activity. ${ }^{39}$ Farmers prefer to plant subsistent crops rather than more labor intensive cash crops. ${ }^{24}$ These costs impede economic growth in malaria endemic countries. It is estimated that the economy of malaria endemic countries is $1.3 \%$ lower than countries without malaria. This estimate seems low. However, when it is taken into consideration that most malaria endemic (GDP) countries are developing with lower gross domestic product, defined as the market value of all final goods and services made within the borders of a country in a year, it is a major factor for endemic countries. ${ }^{40}$

The GDP of a country is often positively correlated with the standard of living. The standard of living of persons living in a malaria endemic area can remain low because of malaria. Malaria disproportionately affects poor people who cannot afford treatment or have limited access to health care. These factors trap families and communities in a downward spiral of poverty. ${ }^{20}$ The economic gap in prosperity between countries with and without malaria grows every year. ${ }^{24}$ It is interesting to note that in almost all countries that have eradicated malaria, there was an immediate economic growth that was faster than neighboring countries. These examples stem from data collected from southern European countries such as Greece, Italy, and Spain. ${ }^{40}$ The richest countries in Africa are free of malaria and located in the northern and southern extremes of the continent. The gross domestic product of malarial countries in 1995 was $\$ 1,526$ compared to $\$ 8,268$ in countries without malaria. ${ }^{40}$

\section{The economic impact of the use of artemisinin}

The era of cheap effective antimalarials has ended but poverty has not. The cost of artemisinin combinational therapies is more than any other antimalarial monotherapy or nonartemisinin combinational therapy. In Tanzania $\$ 2.14$ is spent on malaria control per person per year. This is a huge debt on the country's economy. This number represents $39 \%$ of the health cost and $1.1 \%$ of the country's GDP. ${ }^{39}$ However, in the long run, treating sick people so that they can return to work and school will help the economy. This will translate to economic growth and poverty reduction. In Cameroon, the government subsidizing malarial combination treatment made drug therapy affordable for all people. ${ }^{41}$ This greatly relieved both individuals and the government from the burden of malaria. Overall, this will result in economic development because workers can gain income and economic ventures and tourism will start to flourish.

\section{The economic impact of artemisinin resistance}

As with any antimalarial drug resistance, artemisinin drug resistance has a major impact on health services. With the increase of transmission and drug failures, medical care needs increase. There is a demand for more patient care, diagnostic procedures, and increased costs associated with combination treatments. ${ }^{38}$ So the cycle continues as if the drug never existed. In some countries, chloroquine is still being used even with high failure when alternative drugs such as SP are available. This is a health policy failure, however, policy changes have been significantly associated in terms of cost with retraining health care workers, printing new drug regimens, and stocking new drugs. ${ }^{42}$

Another cost of artemisinin resistance is the increase in morbidity and mortality in the tropical world. ${ }^{43}$ It is estimated that the number of malarial deaths would double with the spread of artemisinin-resistant parasites. People suffer and die because they will not be able to treat them. The economic toll would topple the economy of any developing country providing health care to a great portion of the population of workers and students with no tax revenue in return. The economic prosperity of Thailand and Vietnam, the epicenter of drug resistance to other antimalarials, is threatened by the looming threat of artemisinin resistance. Dr Pascal Ringwald of WHO, who spoke at the ASTMH session in November of 2009 said: "The loss of artemisinin derivatives to resistance could have a devastating effect on health in tropical countries, and would threaten current global efforts to eliminate malaria, as there are very few innovative replacement therapies in the pipeline at the late stage of development". The cost in human morbidity and mortality across the globe would be detrimental to economic development and could reverse any progress that antimalarial efforts have made. ${ }^{44}$ Although the outlook is 
bleak, we are hopeful that some new drug or vaccine will come with billions of dollars put forth in the research effort. It is a matter of a race against time.

\section{Future considerations}

Looking forward, a multipronged approach against malaria, a formidable scourge to worldwide health, can be put in place. Given that artemisinin combination therapies are more expensive than current drugs, several strategies can be undertaken to prevent further emergence of resistance against this valuable antimalarial. First, some countries could switch to SP as an interim measure. This would delay the higher treatment cost of ACTs. However, SP resistance is expected to rise within a few years leading to increased morbidity and mortality. ${ }^{42}$ Second, another source of artemisinin is from partial chemical synthesis. This will help ensure that ACT manufacturers have a consistent, reliable, high quality, and inexpensive supply of the compound. This idea was put forth by the US agency for International Development which commissioned the Institute of Medicine (IOM) in 2001 to ensure broad access to effective antimalarials. The IOM recommended a global subsidy program of ACTs treatment. Therefore, expensive ACTs (cost \$2 USD per treatment) would cost 10 cents per treatment as did CQ and SP. Furthermore, only those patients with a proven case of falciparum malaria would receive ACTs. ${ }^{45}$ Another intervention comes from local and international companies doing business in endemic areas. They are learning to support malaria control thus reducing absenteeism and losses in productivity. ${ }^{24}$ Finally, the continued use of insecticide-treated bednets, and residual spraying should effectively control malaria and slow the spread of drug resistance. Although this multipronged approach maybe effective, they must be implemented and they also must be acceptable to the patients and health care workers. Moreover, patients must adhere to treatment. Some social interventions may be needed to increase successful malaria control.

\section{Disclosure}

The authors report no conflicts of interest in this work.

\section{References}

1. Noji EK. The global resurgence of infectious diseases. Trend Report. 2001;9:223-232.

2. Payne D. Spread of chloroquine resistance in Plasmodium falciparum. Parasitol Today. 1987;3:241-246.

3. White NJ. Qinghaosu (artemisinin): the price of success. Science. 2008;320:330-334.
4. F. ter Kuile, White NJ, Holoway P, Pasvol G, Krishna S. Plasmodium falciparum: in vitro studies of the pharmacodynamic properties of drugs used for the treatment of severe malaria. Exp Parasitol. 1993;76: 85-95.

5. Chen PQ, Li GQ, Guo XB, et al. The infectivity of gametocytes of Plasmodium falciparum from patients treated with artemisinins. Chin Med J. (Engl.) 1994;107:709-711.

6. White NJ, Olliaro P. Artemisinin and derivatives in the treatment of uncomplicated malaria. Med Trop. 1998;58:54-56.

7. Peter W. Session 2: global perspectives of malaria changing pattern of antimalarial drug resistance. J Royal Soc Medicine. (Supp 17) 1989;82:13-16.

8. Eckstein-Ludwig U, Webb R, van Goetham DA, et al. Artemisinins target the SERCA of Plasmodium falciparum. Nature. 2003;424:957-961.

9. Jambou R, Legrand E, Niang M, et al. Resistance of Plasmodium falciparum field isolates to in vitro artemether and point mutations of the SERCA-type PfATPase6. Lancet. 2005;366:1960-1963.

10. Greenhouse B, Slater M, Njama-Meya D, et al. Decreasing efficacy of antimalarial combination therapy in Uganda is explained by decreasing host immunity rather than increasing drug resistance. J Infect Dis. 2009; 199:758-765.

11. Enevold A, Nkya WM, Theisen M, et al. Potential impact of host immunity of malaria treatment outcome in Tanzanian children infected with Plasmodium falciparum. Malar J. 2007;6:153-162.

12. Dondorp AM, Nosten F, Yi P, et al. Artemisinin resistance in Plasmodium falciparum malaria. $N$ Engl J Med. 2009;361:455-67. Erratum in: N Engl J Med. 2009;361:1714.

13. Schönfeld M, Barreto MI, Schunk M, et al. Molecular surveillance of drug-resistance associated mutations of Plasmodium falciparum in south-west Tanzania. Malar J. 2007;15:62.

14. Mboera LE, Makundi EA, Kitua AY. Uncertainty in malaria control in Tanzania: crossroads and challenges for future interventions. Am J Trop Med Hyg. 2007;77:112-118.

15. Mulligan JA, Mandike R, Palmer N, et al. The costs of changing national policy: lessons from malaria treatment policy guidelines in Tanzania. Trop Med Int Health. 2006;11:452-461.

16. Mulligan JA, Mandike R, Palmer N, et al. The costs of changing national policy: lessons from malaria treatment policy guidelines in Tanzania. Trop Med Int Health. 2006;11:452-461.

17. Shretta R, Omumbo J, Rapuoda BA, Snow RW. Using evidence to change antimalarial drug policy in Kenya. Trop Med Int Health. 2000;5:755-764

18. Kamya MR, Bakyaita NN, Talisuna AO, Were WM, Staedke SG. Increasing antimalarial drug resistance in Uganda and revision of the national drug policy. Trop Med Int Health. 2002;7:1031-1041.

19. Kindermans JM, Pecoul B, Perez-Casas C, Boer MD, Berman D, Cox I. Changing national malaria treatment protocols in Africa: what is the cost and who will pay? Case studies: Burundi, Kenya, Rwanda, Tanzania and Uganda. Medicins Sans Frontieres. 2002;1-15.

20. Williams HA, Durrheim DN, Shretta R. The process of changing national malaria treatment policy: lessons from country-level studies. Health Policy Plan. 2004;19:356-370.

21. Mulligan JA, Mandike R, Palmer N, et al. The costs of changing national policy: lessons from malaria treatment policy guidelines in Tanzania. Trop Med Int Health. 2006;11:452-461.

22. World Health Organization. Access to antimalarial medicines: improving the affordability and financing of artemisinin-based combination therapies. Geneva, Switzerland: WHO; 2003:1-15.

23. Amin AA, Zurovac D, Kangwana BB, et al. The challenges of changing national malaria drug policy to artemisinin-based combinations in Kenya. Malar J. 2007;6:72.

24. Williams HA, Durrheim D, Shretta R. The process of changing national malaria treatment policy: lessons from country-level studies. Health Pol Plan. 2004;19:356-370.

25. World Health Organization. Susceptibility of Plasmodium falciparum to antimalarial drugs: report on global monitoring: 1996-2004. (WHO, Geneva). 
26. World Health Organization. Facts on ACTs (Artemisin-based Combination Therapies), January 2006 update. 2006. Available from: http://www. rollbackmalaria.org/cmc_upload/0/000/015/364/RBMInfosheet_9.htm. Accessed Jul 20, 2010.

27. World Health Organization. A global strategy for malaria control. Geneva, Switzerland: WHO; 1993.

28. World Health Organization. World malaria report 2009. Geneva, Switzerland: WHO; 2009.

29. Ashton M, Nguyen DS, Nguyen VH, et al. Artemisinin kinetics and dynamics during oral and rectal treatment of uncomplicated malaria. Clin Pharmacol Ther. 1998;63:482-493.

30. Asimus S, Elsherbiny D, Hai TN, et al. Artemisinin antimalarials moderately affect cytochrome P450 enzyme activity in healthy subjects. Fundam Clin Pharmacol. 2007;21:307-316.

31. World Health Organization. The treatment of malaria. Geneva, Switzerland: WHO; 2006.

32. Dondorp AM, Nosten F, Poravuth D, et al. Reduced in-vivo susceptibility of Plasmodium falciparum to artesunate in Western Cambodia. N Engl J Med. [In press.]

33. Samarasekera U. Countries race to contain resistance to key antimalarial. Lancet. 2009;374:277-280.

34. World Health Organization. World malaria report 2005. Geneva, Switzerland: WHO; 2005.

35. Fofana B, Dijimde AA, Sagara I, et al. Impact of artemisinin-based combination therapy on malaria transmission in Mali. 2009 (MIM16762172 www.minmalaria.org).

36. White N, Nosten F, Looareesuwan S, et al. Averting a malaria disaster. Lancet. 1999;353:1965-1967.
37. Dondorp AM, Yeung S, White L, et al. Artemisinin resistance: current status and scenarios for containment. Nat Rev Microbiol. 2010;8:272-280.

38. Maude RJ, Pontavornpinyo W, Saralamba S, et al. The last man standing is the most resistant: eliminating artemisinin-resistant malaria in Cambodia. Malar J. 2009;8:31.

39. World Health Organization. Global malaria control and elimination: report of a meeting on containment of artemisinin tolerance. Geneva, Switzerland: WHO; 2008.

40. Gallup JL, Sachs JD. The economic burden of malaria. Am J Trop Med Hyg. 2001;64:85-96.

41. Mboera LE, Makundi EA, Kitua AY. Uncertainty in malaria control in Tanzania: crossroads and challenges for future interventions. Am JTrop Med Hyg. 2007;77:112-118.

42. Sayang C, Gausseres M, Vernazza-Licht N, Malvy D, Bley D, Millet P. Treatment of malaria from monotherapy to artemisinin-based combination therapy by health professionals in urban health facilities in Yaoundé, central province, Cameroon. Malar J. 2009;28:176.

43. Sayang C, Gausseres M, Vernazza-Licht N, Malvy D, Bley D, Millet P. Treatment of malaria from monotherapy to artemisinin-based combination therapy by health professionals in urban health facilities in Yaoundé, central province, Cameroon. Malar J. 2009;8:176.

44. Laxminarayan R. ACT Now or later: the economics of malaria resistance. Resources for the future. 2003. Discussion Paper. 3-51.

45. Bjorkman A, Bhattarai A. Public health impact of drug resistant Plasmodium falciparum malaria. Acta Trop. 2005;94:163-169.

46. Panosian C. Economic access to effective drugs for falciparum malaria. Clin Infect Dis. 2005;40:713-717.
Infection and Drug Resistance

\section{Publish your work in this journal}

Infection and Drug Resistance is an international, peer-reviewed openaccess journal that focuses on the optimal treatment of infection (bacterial, fungal and viral) and the development and institution of preventive strategies to minimize the development and spread of resistance. The journal is specifically concerned with the epidemiology of antibiotic

\section{Dovepress}

resistance and the mechanisms of resistance development and diffusion in both hospitals and the community. The manuscript management system is completely online and includes a very quick and fair peerreview system, which is all easy to use. Visit http://www.dovepress.com/ testimonials.php to read real quotes from published authors. 